\title{
ESTIMATES FOR LOWER ORDER EIGENVALUES OF A CLAMPED PLATE PROBLEM*
}

\author{
QING-MING CHENG, GUANGYUE HUANG AND GUOXIN WEI
}

\begin{abstract}
For a bounded domain $\Omega$ in a complete Riemannian manifold $M^{n}$, we study estimates for lower order eigenvalues of a clamped plate problem. We obtain universal inequalities for lower order eigenvalues. We would like to remark that our results are sharp.
\end{abstract}

\section{INTRODUCTION}

Let $\Omega$ be a bounded domain in an $n$-dimensional complete Riemannian manifold $M^{n}$. Assume that $\Gamma_{i}$ is the $i^{\text {th }}$ eigenvalue of a clamped plate problem, which describes characteristic vibrations of a clamped plate:

$$
\begin{cases}\Delta^{2} u=\Gamma u, & \text { in } \Omega, \\ u=\frac{\partial u}{\partial \nu}=0, & \text { on } \partial \Omega,\end{cases}
$$

where $\Delta$ is the Laplacian on $M^{n}$ and $\nu$ denotes the outward unit normal to the boundary $\partial \Omega$. It is well known that this problem has a real and discrete spectrum

$$
0<\Gamma_{1} \leq \Gamma_{2} \leq \cdots \leq \Gamma_{k} \leq \cdots \nearrow+\infty,
$$

where each $\Gamma_{i}$ has finite multiplicity which is repeated according to its multiplicity.

In this paper, we do not introduce results on universal inequalities for higher order eigenvalues of the clamped plate problem. The readers who are interested in it can see the references [3], [4], [5], 7], [8] and [10]. Since for lower order eigenvalues of the clamped plate problem, one can obtain better universal inequalities for eigenvalues. We will focus our mind on the investigation of lower order eigenvalues of the clamped plate problem.

When $M^{n}$ is an $n$-dimensional Euclidean space, for lower order eigenvalues of the clamped plate problem (1.1), Ashbaugh [1] announced the following two universal inequalities without proofs. Cheng, Ichikawa and Mametsuka [4] have given their proofs.

$$
\begin{aligned}
& \sum_{i=1}^{n}\left(\Gamma_{i+1}^{\frac{1}{2}}-\Gamma_{1}^{\frac{1}{2}}\right) \leq 4 \Gamma_{1}^{\frac{1}{2}}, \\
& \sum_{i=1}^{n}\left(\Gamma_{i+1}-\Gamma_{1}\right) \leq 24 \Gamma_{1} .
\end{aligned}
$$

2001 Mathematics Subject Classification: 35P15, 58G25, 53C42.

Key words and phrases: estimates for eigenvalues, clamped plate problem.

* Research partially Supported by a Grant-in-Aid for Scientific Research from JSPS. 
When $M^{n}$ is a general complete Riemannian manifold other than the Euclidean space, it is natural to consider the following problem:

Problem. Let $M^{n}$ be an $n$-dimensional complete Riemannian manifold and $\Omega$ a bounded domain in $M^{n}$. Whether can one obtain a universal inequality for lower order eigenvalues, which are analogous to (1.2), of the clamped plate problem?

In this paper, we will answer the problem and prove the following results:

Theorem 1. Let $\Omega$ be a bounded domain in an n-dimensional complete Riemannian manifold $M^{n}$. For the lower order eigenvalues of the clamped plate problem:

$$
\begin{cases}\Delta^{2} u=\Gamma u, & \text { in } \Omega \\ u=\frac{\partial u}{\partial \nu}=0, & \text { on } \partial \Omega\end{cases}
$$

we have

$$
\sum_{i=1}^{n}\left(\Gamma_{i+1}-\Gamma_{1}\right)^{\frac{1}{2}} \leq\left(4 \Gamma_{1}^{\frac{1}{2}}+n^{2} H_{0}^{2}\right)^{\frac{1}{2}}\left\{(2 n+4) \Gamma_{1}^{\frac{1}{2}}+n^{2} H_{0}^{2}\right\}^{\frac{1}{2}},
$$

where $H_{0}^{2}$ is a nonnegative constant which only depends on $M^{n}$ and $\Omega$.

Corollary 1. Under the assumption of the theorem 1, we have

$$
\sum_{i=1}^{n}\left\{\left(\Gamma_{i+1}-\Gamma_{1}\right)^{\frac{1}{2}}-\Gamma_{1}^{\frac{1}{2}}\right\} \leq 4 \Gamma_{1}^{\frac{1}{2}}+n^{2} H_{0}^{2}
$$

Corollary 2. When $M^{n}$ is an $n$-dimensional complete minimal submanifold in a Euclidean space, we have

$$
\sum_{i=1}^{n}\left(\Gamma_{i+1}-\Gamma_{1}\right)^{\frac{1}{2}} \leq\left\{8(n+2) \Gamma_{1}\right\}^{\frac{1}{2}}
$$

Corollary 3. When $M^{n}$ is an n-dimensional unit sphere, we have

$$
\sum_{i=1}^{n}\left(\Gamma_{i+1}-\Gamma_{1}\right)^{\frac{1}{2}} \leq\left(4 \Gamma_{1}^{\frac{1}{2}}+n^{2}\right)^{\frac{1}{2}}\left\{(2 n+4) \Gamma_{1}^{\frac{1}{2}}+n^{2}\right\}^{\frac{1}{2}} .
$$

Remark 1. For the unit sphere $S^{n}(1)$, by taking $\Omega=S^{n}(1)$, we know $\Gamma_{1}=0$ and $\Gamma_{2}=\cdots=\Gamma_{n+1}=n^{2}$. Hence, our inequalities become equalities. Thus, our results are sharp.

Remark 2. After the first author and the third author have proved the above results, the second author tells them that he has also proved the same results. Hence, the authors decide to write this joint paper together. 


\section{PRELIMinaries}

In order to prove Theorem 1, we need the following Nash's theorem.

Nash's theorem. Each complete Riemannian manifold $M^{n}$ can be isometrically immersed into a Euclidian space $\mathbb{R}^{N}$.

Assume that $M^{n}$ is an $n$-dimensional isometrically immersed submanifold in $\mathbb{R}^{N}$. Let $\Omega \subset M^{n}$ be a bounded domain of $M^{n}$ and $p \in \Omega$. Let $\left(x^{1}, \cdots, x^{n}\right)$ be a local coordinate system in a neighborhood $U$ of $p \in M$. Let $\mathbf{y}$ be the position vector of $p$ in $\mathbb{R}^{N}$, which is defined by

$$
\mathbf{y}=\left(y^{1}\left(x^{1}, \cdots, x^{n}\right), \cdots, y^{N}\left(x^{1}, \cdots, x^{n}\right)\right) .
$$

Since $M^{n}$ is isometrically immersed in $\mathbb{R}^{N}$, we have

$$
g_{i j}=g\left(\frac{\partial}{\partial x^{i}}, \frac{\partial}{\partial x^{j}}\right)=<\sum_{\alpha=1}^{N} \frac{\partial y^{\alpha}}{\partial x^{i}} \frac{\partial}{\partial y^{\alpha}}, \sum_{\beta=1}^{N} \frac{\partial y^{\beta}}{\partial x^{i}} \frac{\partial}{\partial y^{\beta}}>=\sum_{\alpha=1}^{N} \frac{\partial y^{\alpha}}{\partial x^{i}} \frac{\partial y^{\alpha}}{\partial x^{j}},
$$

where $g$ denotes the induced metric of $M^{n}$ from $\mathbb{R}^{N}$ and $<,>$ is the standard inner product in $\mathbb{R}^{N}$. The following lemma can be found in [2].

Lemma. For any function $u \in C^{\infty}\left(M^{n}\right)$, we have

$$
\begin{gathered}
\sum_{\alpha=1}^{N}\left(g\left(\nabla y^{\alpha}, \nabla u\right)\right)^{2}=|\nabla u|^{2}, \\
\sum_{\alpha=1}^{N} g\left(\nabla y^{\alpha}, \nabla y^{\alpha}\right)=\sum_{\alpha=1}^{N}\left|\nabla y^{\alpha}\right|^{2}=n, \\
\sum_{\alpha=1}^{N}\left(\Delta y^{\alpha}\right)^{2}=n^{2}|H|^{2}, \\
\sum_{\alpha=1}^{N} \Delta y^{\alpha} \nabla y^{\alpha}=0,
\end{gathered}
$$

where $\nabla$ denotes the gradient operator on $M^{n}$ and $|H|$ is the mean curvature of $M^{n}$.

\section{Proofs of Results}

Proof of Theorem 1. Since $M^{n}$ is a complete Riemannian manifold, Nash's theorem implies that there exists an isometric immersion from $M^{n}$ into a Euclidean space $\mathbb{R}^{N}$. Thus, $M^{n}$ can be considered as an $n$-dimensional complete isometrically immersed submanifold in $\mathbb{R}^{N}$.

Let $u_{i}$ be an eigenfunction corresponding to eigenvalue $\Gamma_{i}$ such that $\left\{u_{i}\right\}_{i \in \mathbf{N}}$ becomes an orthonormal basis of $L^{2}(\Omega)$, that is,

$$
\int_{\Omega} u_{i} u_{j}=\delta_{i j}, \quad \forall i, j \in \mathbf{N} .
$$


We define an $N \times N$-matrix $B$ as follows:

$$
B:=\left(b_{\alpha \beta}\right)
$$

where $b_{\alpha \beta}=\int_{\Omega} y^{\alpha} u_{1} u_{\beta+1}$ and $\mathbf{y}=\left(y^{\alpha}\right)$ is the position vector of the immersion in $\mathbb{R}^{N}$. Using the orthogonalization of Gram and Schmidt, we know that there exist an upper triangle matrix $R=\left(R_{\alpha \beta}\right)$ and an orthogonal matrix $Q=\left(q_{\alpha \beta}\right)$ such that $R=Q B$, i.e.,

$$
R_{\alpha \beta}=\sum_{\gamma=1}^{N} q_{\alpha \gamma} b_{\gamma \beta}=\int_{\Omega} \sum_{\gamma=1}^{N} q_{\alpha \gamma} y^{\gamma} u_{1} u_{\beta+1}=0, \quad 1 \leq \beta<\alpha \leq N .
$$

Defining $g^{\alpha}=\sum_{\gamma=1}^{N} q_{\alpha \gamma} y^{\gamma}$, we get

$$
\int_{\Omega} g^{\alpha} u_{1} u_{\beta+1}=\int_{\Omega} \sum_{\gamma=1}^{N} q_{\alpha \gamma} y^{\gamma} u_{1} u_{\beta+1}=0, \quad 1 \leq \beta<\alpha \leq N .
$$

We put

$$
\psi_{\alpha}:=\left(g^{\alpha}-a^{\alpha}\right) u_{1}, \quad a^{\alpha}:=\int_{\Omega} g^{\alpha} u_{1}^{2}, \quad 1 \leq \alpha \leq N,
$$

then it follows that

$$
\int_{\Omega} \psi_{\alpha} u_{\beta+1}=0, \quad 0 \leq \beta<\alpha \leq N .
$$

Thus, $\psi_{\alpha}, 1 \leq \alpha \leq N$, are trial functions. From the Rayleigh-Ritz inequality, we have

$$
\Gamma_{\alpha+1} \leq \frac{\int_{\Omega} \psi_{\alpha} \Delta^{2} \psi_{\alpha}}{\int_{\Omega} \psi_{\alpha}^{2}}, \quad 1 \leq \alpha \leq N
$$

By a direct calculation, we have

$$
\begin{aligned}
\int_{\Omega} \psi_{\alpha} \Delta^{2} \psi_{\alpha} & =\int_{\Omega} \psi_{\alpha} \Delta^{2}\left(g^{\alpha} u_{1}-a^{\alpha} u_{1}\right) \\
& =\int_{\Omega} \psi_{\alpha}\left\{u_{1} \Delta^{2} g^{\alpha}+2 \nabla\left(\Delta g^{\alpha}\right) \cdot \nabla u_{1}+2 \Delta g^{\alpha} \Delta u_{1}\right. \\
& \left.+2 \Delta\left(\nabla g^{\alpha} \cdot \nabla u_{1}\right)+2 \nabla g^{\alpha} \cdot \nabla\left(\Delta u_{1}\right)+\Gamma_{1} g^{\alpha} u_{1}\right\} .
\end{aligned}
$$

Then by (3.4) and (3.5), we conclude

$$
\left(\Gamma_{\alpha+1}-\Gamma_{1}\right)\left\|\psi_{\alpha}\right\|^{2} \leq \int_{\Omega} r_{\alpha} \psi_{\alpha}:=\omega_{\alpha}, \quad 1 \leq \alpha \leq N
$$

where

$$
\begin{aligned}
r_{\alpha} & =\Delta^{2}\left(g^{\alpha} u_{1}\right)-a^{\alpha} \Delta^{2} u_{1} \\
& =u_{1} \Delta^{2} g^{\alpha}+2 \nabla\left(\Delta g^{\alpha}\right) \cdot \nabla u_{1}+2 \Delta g^{\alpha} \Delta u_{1}+2 \Delta\left(\nabla g^{\alpha} \cdot \nabla u_{1}\right)+2 \nabla g^{\alpha} \cdot \nabla\left(\Delta u_{1}\right) .
\end{aligned}
$$

By making use of Stokes' formula, it is easy to get

$$
\int_{\Omega} r_{\alpha} a^{\alpha} u_{1}=0
$$


and

$$
\omega_{\alpha}=\int_{\Omega} r_{\alpha} \psi_{\alpha}=\int_{\Omega} r_{\alpha}\left(g^{\alpha} u_{1}-a^{\alpha} u_{1}\right)=\int_{\Omega} r_{\alpha} g^{\alpha} u_{1} .
$$

We also obtain the following equations from Stokes' theorem

$$
\begin{gathered}
2 \int_{\Omega} g^{\alpha} u_{1} \nabla\left(\Delta g^{\alpha}\right) \cdot \nabla u_{1}=\int_{\Omega}\left\{2 u_{1} \Delta g^{\alpha} \nabla u_{1} \cdot \nabla g^{\alpha}+u_{1}^{2}\left(\Delta g^{\alpha}\right)^{2}-g^{\alpha} u_{1}^{2} \Delta^{2} g^{\alpha}\right\}, \\
2 \int_{\Omega} g^{\alpha} u_{1} \Delta\left(\nabla g^{\alpha} \cdot \nabla u_{1}\right)=\int_{\Omega}\left\{2 u_{1} \Delta g^{\alpha} \nabla g^{\alpha} \cdot \nabla u_{1}+4\left(\nabla g^{\alpha} \cdot \nabla u_{1}\right)^{2}+2 g^{\alpha} \Delta u_{1} \nabla g^{\alpha} \cdot \nabla u_{1}\right\}, \\
2 \int_{\Omega} g^{\alpha} u_{1} \nabla g^{\alpha} \cdot \nabla\left(\Delta u_{1}\right)=-2 \int_{\Omega}\left\{\left|\nabla g^{\alpha}\right|^{2} u_{1} \Delta u_{1}+g^{\alpha} \Delta u_{1} \nabla g^{\alpha} \cdot \nabla u_{1}+g^{\alpha} \Delta g^{\alpha} u_{1} \Delta u_{1}\right\} .
\end{gathered}
$$

Consequently, we get

$$
\begin{aligned}
\omega_{\alpha} & =\int_{\Omega}\left\{\left(\Delta g^{\alpha}\right)^{2} u_{1}^{2}+4\left(\nabla g^{\alpha} \cdot \nabla u_{1}\right)^{2}-2\left|\nabla g^{\alpha}\right|^{2} u_{1} \Delta u_{1}+4 u_{1} \Delta g^{\alpha} \nabla g^{\alpha} \cdot \nabla u_{1}\right\} \\
& =\left\|u_{1} \Delta g^{\alpha}+2 \nabla g^{\alpha} \cdot \nabla u_{1}\right\|^{2}-2 \int_{\Omega}\left|\nabla g^{\alpha}\right|^{2} u_{1} \Delta u_{1} .
\end{aligned}
$$

(3.7) and (3.8) imply

$$
\left(\Gamma_{\alpha+1}-\Gamma_{1}\right)\left\|\psi_{\alpha}\right\|^{2} \leq\left\|u_{1} \Delta g^{\alpha}+2 \nabla g^{\alpha} \cdot \nabla u_{1}\right\|^{2}-2 \int_{\Omega}\left|\nabla g^{\alpha}\right|^{2} u_{1} \Delta u_{1}, \quad 1 \leq \alpha \leq N .
$$

On the other hand,

$$
\begin{aligned}
& \int_{\Omega} \psi_{\alpha}\left(u_{1} \Delta g^{\alpha}+2 \nabla u_{1} \cdot \nabla g^{\alpha}\right) \\
= & \int_{\Omega}\left(g^{\alpha} u_{1}-u_{1} a^{\alpha}\right)\left(u_{1} \Delta g^{\alpha}+2 \nabla u_{1} \cdot \nabla g^{\alpha}\right) \\
= & \int_{\Omega} g^{\alpha} u_{1}\left(u_{1} \Delta g^{\alpha}+2 \nabla u_{1} \cdot \nabla g^{\alpha}\right) \\
= & \int_{\Omega} g^{\alpha} u_{1}^{2} \Delta g^{\alpha}+\frac{1}{2} \nabla u_{1}^{2} \cdot \nabla\left(g^{\alpha}\right)^{2} .
\end{aligned}
$$

By using of Stokes' formula, we know

$$
\int_{\Omega} g^{\alpha} u_{1}^{2} \Delta g^{\alpha}=-\int_{\Omega}\left|u_{1} \nabla g^{\alpha}\right|^{2}-\frac{1}{2} \int_{\Omega} \nabla u_{1}^{2} \cdot \nabla\left(g^{\alpha}\right)^{2} .
$$

Substituting (3.11) into (3.10), we infer

$$
-\int_{\Omega} \psi_{\alpha}\left(u_{1} \Delta g^{\alpha}+2 \nabla u_{1} \cdot \nabla g^{\alpha}\right)=\int_{\Omega}\left|u_{1} \nabla g^{\alpha}\right|^{2} .
$$


From (3.12) and (3.9), we have

$$
\begin{aligned}
& \left(\Gamma_{\alpha+1}-\Gamma_{1}\right)^{\frac{1}{2}} \int_{\Omega}\left|u_{1} \nabla g^{\alpha}\right|^{2} \\
& =-\left(\Gamma_{\alpha+1}-\Gamma_{1}\right)^{\frac{1}{2}} \int_{\Omega} \psi_{\alpha}\left(u_{1} \Delta g^{\alpha}+2 \nabla u_{1} \cdot \nabla g^{\alpha}\right) \\
& \leq \frac{\delta}{2}\left(\Gamma_{\alpha+1}-\Gamma_{1}\right)\left\|\psi_{\alpha}\right\|^{2}+\frac{1}{2 \delta}\left\|u_{1} \Delta g^{\alpha}+2 \nabla u_{1} \cdot \nabla g^{\alpha}\right\|^{2} \\
& \leq\left(\frac{\delta}{2}+\frac{1}{2 \delta}\right)\left\|u_{1} \Delta g^{\alpha}+2 \nabla u_{1} \cdot \nabla g^{\alpha}\right\|^{2}-\delta \int_{\Omega}\left|\nabla g^{\alpha}\right|^{2} u_{1} \Delta u_{1} .
\end{aligned}
$$

According to the lemma in the section 2 and the definition of $g^{\alpha}$, we then have

$$
\begin{aligned}
& \sum_{\alpha=1}^{N}\left\|u_{1} \Delta g^{\alpha}+2 \nabla g^{\alpha} \cdot \nabla u_{1}\right\|^{2} \\
= & \sum_{\alpha=1}^{N} \int_{\Omega}\left\{u_{1}^{2}\left(\Delta g^{\alpha}\right)^{2}+4\left(\nabla u_{1} \cdot \nabla g^{\alpha}\right)^{2}+2\left(\Delta g^{\alpha} \nabla g^{\alpha}\right) \cdot \nabla u_{1}^{2}\right\} \\
= & n^{2} \int_{\Omega}|H|^{2} u_{1}^{2}+4 \int_{\Omega}\left|\nabla u_{1}\right|^{2} \\
\leq & 4 \Gamma_{1}^{\frac{1}{2}}+n^{2} \sup _{\Omega}|H|^{2} .
\end{aligned}
$$

For any point $p$, by a transformation of coordinates if necessary, we have, for any $\alpha$,

$$
\left|\nabla g^{\alpha}\right|^{2}=g\left(\nabla g^{\alpha}, \nabla g^{\alpha}\right) \leq 1
$$

From (3.15), we infer

$$
\begin{aligned}
& \sum_{\alpha=1}^{N}\left(\Gamma_{\alpha+1}-\Gamma_{1}\right)^{\frac{1}{2}}\left|\nabla g^{\alpha}\right|^{2} \\
& \geq \sum_{i=1}^{n}\left(\Gamma_{i+1}-\Gamma_{1}\right)^{\frac{1}{2}}\left|\nabla g^{i}\right|^{2}+\left(\Gamma_{n+1}-\Gamma_{1}\right)^{\frac{1}{2}} \sum_{A=n+1}^{N}\left|\nabla g^{A}\right|^{2} \\
& =\sum_{i=1}^{n}\left(\Gamma_{i+1}-\Gamma_{1}\right)^{\frac{1}{2}}\left|\nabla g^{i}\right|^{2}+\left(\Gamma_{n+1}-\Gamma_{1}\right)^{\frac{1}{2}}\left(n-\sum_{j=1}^{n}\left|\nabla g^{j}\right|^{2}\right) \\
& =\sum_{i=1}^{n}\left(\Gamma_{i+1}-\Gamma_{1}\right)^{\frac{1}{2}}\left|\nabla g^{i}\right|^{2}+\left(\Gamma_{n+1}-\Gamma_{1}\right)^{\frac{1}{2}} \sum_{j=1}^{n}\left(1-\left|\nabla g^{j}\right|^{2}\right) \\
& \geq \sum_{i=1}^{n}\left(\Gamma_{i+1}-\Gamma_{1}\right)^{\frac{1}{2}}\left|\nabla g^{i}\right|^{2}+\sum_{j=1}^{n}\left(\Gamma_{j+1}-\Gamma_{1}\right)^{\frac{1}{2}}\left(1-\left|\nabla g^{j}\right|^{2}\right) \\
& =\sum_{j=1}^{n}\left(\Gamma_{j+1}-\Gamma_{1}\right)^{\frac{1}{2}} .
\end{aligned}
$$


For (3.13), taking sum on $\alpha$ from 1 to $N$ and using of (3.14) and the above inequality, we obtain

$$
\sum_{i=1}^{n}\left(\Gamma_{i+1}-\Gamma_{1}\right)^{\frac{1}{2}} \leq\left(\frac{\delta}{2}+\frac{1}{2 \delta}\right)\left(4 \Gamma_{1}^{\frac{1}{2}}+n^{2} \sup _{\Omega}|H|^{2}\right)+n \delta \Gamma_{1}^{\frac{1}{2}}
$$

Taking

$$
\delta=\sqrt{\frac{4 \Gamma_{1}^{\frac{1}{2}}+n^{2} \sup _{\Omega}|H|^{2}}{4 \Gamma_{1}^{\frac{1}{2}}+n^{2} \sup _{\Omega}|H|^{2}+2 n \Gamma_{1}^{\frac{1}{2}}}},
$$

we obtain

$$
\sum_{i=1}^{n}\left(\Gamma_{i+1}-\Gamma_{1}\right)^{\frac{1}{2}} \leq\left(4 \Gamma_{1}^{\frac{1}{2}}+n^{2} \sup _{\Omega}|H|^{2}\right)^{\frac{1}{2}}\left\{(2 n+4) \Gamma_{1}^{\frac{1}{2}}+n^{2} \sup _{\Omega}|H|^{2}\right\}^{\frac{1}{2}} .
$$

Since the spectrum of the clamped plate problem is an invariant of isometries, we know that (3.16) holds for any isometric immersion from $M^{n}$ into a Euclidean space.

Now we define $\Phi$ as follows:

$$
\Phi:=\{\psi \mid \psi \text { is an isometric immersion from } M \text { into a Euclidian space }\} .
$$

Defining

$$
H_{0}^{2}:=\inf _{\psi \in \Phi} \sup _{\Omega}|H|^{2}
$$

we obtain

$$
\sum_{i=1}^{n}\left(\Gamma_{i+1}-\Gamma_{1}\right)^{\frac{1}{2}} \leq\left(4 \Gamma_{1}^{\frac{1}{2}}+n^{2} H_{0}^{2}\right)^{\frac{1}{2}}\left\{(2 n+4) \Gamma_{1}^{\frac{1}{2}}+n^{2} H_{0}^{2}\right\}^{\frac{1}{2}} .
$$

This completes the proof of Theorem 1.

Proof of Corollary 1. Since

$$
\left(4 \Gamma_{1}^{\frac{1}{2}}+n^{2} H_{0}^{2}\right)^{\frac{1}{2}}\left\{(2 n+4) \Gamma_{1}^{\frac{1}{2}}+n^{2} H_{0}^{2}\right\}^{\frac{1}{2}} \leq 4 \Gamma_{1}^{\frac{1}{2}}+n^{2} H_{0}^{2}+n \Gamma_{1}^{\frac{1}{2}} .
$$

from (3.17), we then obtain

$$
\sum_{i=1}^{n}\left\{\left(\Gamma_{i+1}-\Gamma_{1}\right)^{\frac{1}{2}}-\Gamma_{1}^{\frac{1}{2}}\right\} \leq 4 \Gamma_{1}^{\frac{1}{2}}+n^{2} H_{0}^{2}
$$

This finishes the proof of Corollary 1.

Proof of Corollary 2. For a complete minimal submanifold in a Euclidean space, we have $|H|=0$. From the proof of the theorem 1, the corollary 2 is clear.

Proof of Corollary 3. Since an $n$-dimensional unit sphere can be seen as a compact hypersurface with constant mean curvature 1 in the Euclidean space $\mathbb{R}^{n+1}$, we have $|H|=1$. From the proof of the theorem 1, the corollary 3 is obvious. 
QING-MING CHENG, GUANGYUE HUANG AND GUOXIN WEI

\section{REFERENCES}

[1] M. S. Ashbaugh, Isoperimetric and universal inequalities for eigenvalues, in spectral theory and geometry (Edinburgh, 1998, E. B. Davies and Yu Safarov, eds.), London Math. Soc. Lecture Notes, 273 (1999),95-139.

[2] D. G. Chen and Q. -M. Cheng, Extrinsic estimates for eigenvalues of the Laplace operator, J. Math. Soc. Japan 60 (2008), 325-339.

[3] Z. C. Chen and C. L. Qian, Estimates for discrete spectrum of Laplacian operator with any order, J.China Univ. Sci. Tech. 20 (1990), 259-266.

[4] Q. -M. Cheng, T. Ichikawa and S. Mametsuka, Inequalities for eigenvalues of Laplacian with any order, to appear in Commun. Contemp. Math., 2009.

[5] Q. -M. Cheng, T. Ichikawa and S. Mametsuka, Estimates for eigenvalues of a clamped plate problem on Riemannian manifolds, to appear in J. Math. Soc. Japan.

[6] Q. -M. Cheng and H. C. Yang, Inequalities for eigenvalues of a clamped plate problem, Trans. Amer. Math. Soc. 358 (2006), 2625-2635.

[7] S. M. Hook, Domain independent upper bounds for eigenvalues of elliptic operator, Trans. Amer. Math. Soc., 318(1990), 615-642.

[8] L. E. Payne, G. Pólya and H. F. Weinberger, On the ratio of consecutive eigenvalues, J. Math. and Phys. 35 (1956), 289-298.

[9] H. Sun, Q. -M. Cheng and H. C. Yang, Lower order eigenvalues of Dirichlet Laplacian, Manuscripta Math. 125 (2008), 139-156.

[10] Q. L. Wang, C. Y. Xia, Universal bounds for eigenvalues of the biharmonic operator on Riemannian manifolds, J. Funct. Anal. 245 (2007), 334-352.

\section{Qing-Ming Cheng}

Department of Mathematics

Faculty of Science and Engineering

Saga University

840-8502, Saga

Japan

E-mail: cheng@ms.saga-u.ac.jp
Guangyue Huang

Department of Mathematics

Henan Normal University

453007, Xinxiang

People's Republic of China

E-mail: hgy@henannu.edu.cn

\section{Guoxin Wei}

Department of Mathematics

Faculty of Science and Engineering

Saga University

840-8502, Saga

Japan

E-mail:wei@ms.saga-u.ac.jp 